\title{
Uso y abuso de dispositivos móviles y su rol en el desarrollo de trastornos del sueño en adolescentes
}

\author{
DOI 10.5377/alerta.v5i1.11247 \\ Daniela Carolina Iraheta Lara ${ }^{4}$, Héctor Elías Menjívar Saravia ${ }^{5}$ \\ 1-5. Universidad Dr. José Matías Delgado, San Salvador, El Salvador \\ ${ }^{*}$ Correspondencia \\ $\square$ jcelisinfante@gmail.com \\ 1. (D) 0000-0003-4077-090X \\ 2. (1) $0000-0002-5775-508 \mathrm{X}$ \\ 3. (D) $0000-0001-7052-262 \mathrm{X}$ \\ 4. (1) 0000-0001-9687-9037 \\ 5. (1) 0000-0002-9461-9204
}

José Antonio Celis Infante ${ }^{1 *}$, Marcela Alejandra Benavides Romero ${ }^{2}$, Paola Marcela del Cid Amaya ${ }^{3}$,

G

ACCESO ABIERTO

Use and abuse of mobile devices and their role in the development of sleep disorders in adolescents

Citación recomendada: Celis Infante JA, Benavides Romero MA, del Cid Amaya PM, Iraheta Lara DC, Menjívar Saravia HE. Uso y abuso de dispositivos móviles y su rol en el desarrollo de trastornos del sueño en adolescentes. Alerta. 2022;5(1):50-56. DOI 10.5377/ alerta.v5i1.11247

Recibido:

04 de junio de 2021

Aceptado:

31 de agosto 2021.

Publicado:

27 de enero de 2022.

Contribución de autoría: JACl': introducción, trastornos del sueño y conclusiones. MABR2: resumen, introducción, asociación entre el uso de las TIC y trastornos del sueño y conclusiones. PMDCA3: resumen, introducción, pandemia y conclusiones. DCIL ${ }^{4}$ : introducción, perfiles del sueño y conclusiones. HEMS : introducción, insomnio, trastornos del sueño y conclusiones.

\section{Conflicto de intereses:}

Los autores declaran que no existe conflicto de interés.

\begin{abstract}
Resumen
Las tecnologías de información y comunicación suponen un avance para la sociedad. Hoy en día, los dispositivos móviles proveen servicios convenientes para la cotidianidad, facilitando la comunicación, el ocio y las relaciones interpersonales, sin embargo, se describe que su uso excesivo influye en la aparición de síntomas como ansiedad, comportamientos adictivos y alteraciones del sueño. De todos los usuarios de estas tecnologías, los adolescentes son un grupo especialmente afectado, debido al fácil acceso a estas desde edades muy tempranas y la consecuente integración a su vida diaria. Por lo que se pretende determinar el papel del uso de dispositivos móviles en el desarrollo de trastornos del sueño en adolescentes. Pese a sus efectos deletéreos, se ha observado que el uso de estas tecnologías puede ocasionar sensación de bienestar y apoyo social en algunos usuarios, particularmente cuando promueve la interacción con sus pares. El uso excesivo de las tecnologías, en especial horas antes de dormir, se relaciona con un patrón de sueño anómalo, se ha identificado que los trastornos del sueño más prevalentes en los usuarios son el insomnio y la somnolencia diurna excesiva.

Palabras clave

Adolescentes, disomnias, tecnologías de la información, sueño, internet
\end{abstract}

\begin{abstract}
Information and communication technologies represent an advance for society. Nowadays, mobile devices provide convenient services for everyday life, facilitating communication triggers, leisure and interpersonal relationships, however, it is described that their excessive use influences the appearance of symptoms such as anxiety, addictive behaviors and sleep disturbances. Adolescents are a particularly affected group, with easy access to this technology from an early age and its consequent integration into their daily life. Therefore, it is intended to determine the role played by the use and abuse of mobile devices in the development of sleep disorders in adolescents. Despite their deleterious effects, it has been observed that the use of these technologies can cause a feeling of well-being and social support in some users, particularly when it promotes interaction with their peers. The excessive use of these technologies, especially hours before sleeping, is related to an abnormal sleep patterns, it has been identified that the most prevalent sleep disorders in users are insomnia and excessive daytime sleepiness.
\end{abstract}

Keywords

Teenagers, dyssomnias, information technology, sleep, internet

\section{Introducción}

Actualmente, los dispositivos móviles proveen servicios convenientes para la cotidianidad, facilitando la comunicación y las relaciones interpersonales de aproximadamente 2,87 billones de usuarios en 2020'. La población adolescente tiene como hábito el uso de las tecnologías de la información y de la comunicación (TIC) desde edades tempranas, lo que les permite desarrollar amistades y encontrar un espacio independiente de los padres, pero su uso excesivo se asocia a la aparición de comportamientos adictivos en los usuarios, según los resultados de Cha et al.?. 
El contexto actual de confinamiento le otorga a las TIC un rol importante en la socialización, incrementando su uso. El estudio en línea también aumenta el tiempo de exposición a los dispositivos, afectando la calidad del sueño ${ }^{3,4}$. El uso problemático de las TIC en adolescentes se manifiesta con síntomas de dependencia y abstinencia, ocasionando además, ausentismo escolar, agresividad, ansiedad, alteraciones del sueño o bajo rendimiento académico 5 .

En los Estados Unidos de América, Hale L, et al., en 2018 reportaron el 51 \% de personas con impactos significativos en el estado de ánimo, el funcionamiento diario y las relaciones interpersonales ${ }^{6}$. Además, el uso de internet como entretenimiento promueve un patrón de sueño más corto y disminuye su calidad?.

Durante la adolescencia se producen cambios que requieren de buenos hábitos de sueño, por lo que se necesitan entre 8 y 10 horas para un desarrollo psicofisiológico adecuado ${ }^{8}$. Según la Asociación Estadounidense de Psiquiatría (American Psychiatry Association, APA), los trastornos del sueño se caracterizan por problemas en la calidad y la cantidad del sueño?

El uso de las redes sociales se relaciona con efectos negativos en el sueño, las relaciones interpersonales y el desempeño laboral y escolarro . Se considera que las TIC desplazan o interrumpen el tiempo de sueño, proveen contenido estimulante y alteran el ciclo circadiano ${ }^{11}$. Por otro lado, las alteraciones en los patrones de sueño de los adolescentes se relacionan con un incremento de alteraciones de ánimo, ansiedad, abuso de sustancias, problemas de comportamiento e ideaciones suicidas ${ }^{12}$. Por lo anterior se pretende determinar el rol ejercido por el uso y abuso de dispositivos móviles en el desarrollo de trastornos del sueño en adolescentes.

\section{Desarrollo}

\section{Perfil de sueño y trastornos del sueño en adolescentes}

El sueño es un proceso fisiológico y una necesidad biológica de los seres humanos que les permite lograr un estado adecuado de salud. Consiste en una etapa relativa de inactividad física en la que se restablecen las funciones psicológicas, neuroendocrinas, gástricas e intestinales, que son vitales para un pleno rendimiento $y$, a su vez, la persona se encuentra en un estado de descanso y recuperación ${ }^{13}$.

La calidad de sueño depende de varios factores relacionados con la persona, las etapas de su ciclo del sueño, el consumo de medicamentos, estimulantes como la cafeína y teína, ejercicios, entorno laboral o algunas enfermedades, además de otros aspectos ambientales, tales como el uso de dispositivos tecnológicos. El índice de calidad de sueño de Pittsburgh (ICSP), es un instrumento estándar para la medición del sueño mediante el cual se analizan sus diferentes factores determinantes. Estos se agrupan en siete componentes: calidad, latencia, duración, eficiencia, perturbaciones del sueño, uso de medicación para dormir y disfunción diurna. Tiene una puntuación de 0 a 21, donde a mayor puntuación, peor la calidad de sueño ${ }^{14}$.

Según un estudio realizado por la Universidad de Granada, España 2002, en el cual la cantidad del sueño se midió en horas, se consideró que si se duerme diariamente 5,5 horas o menos, es un patrón de sueño corto, 9 horas o más es un patrón de sueño largo y entre 7 y 8 horas es un patrón de sueño intermedio ${ }^{15}$.

En cuanto a los trastornos del sueño, según el Manual diagnóstico y estadístico de transtornos mentales (DSM-V), el insomnio se define como una predominante insatisfacción por la cantidad o la calidad del sueño, que se puede presentar como dificultad para conciliar el sueño, mantenerlo o despertar pronto por la mañana y ser incapaz de volver a dormir; esta sintomatología se presenta en al menos tres días a la semana durante un periodo de tiempo igual o mayor a tres meses ${ }^{16}$.

Actualmente, la prevalencia de insomnio en adolescentes de 16-18 años corresponde al $18,5 \%$ a predominio del sexo femenino $(23,8 \%)^{17}$. No se conoce la causa exacta del por qué las mujeres presentan un mayor porcentaje, pero se cree que el inicio de la menstruación es un evento que aumenta el riesgo de desarrollar esta patología ${ }^{18}$.

El impacto del insomnio sobre la vida normal del adolescente se presenta de diferentes formas, tales como somnolencia diurna, alteraciones en la atención durante el día y disminución del índice de calidad de vida relacionado con la salud ( $\mathrm{HRQOL}$, por sus siglas en inglés) ${ }^{19}$. Aparte de las consecuencias antes expuestas, existe evidencia que indica que el insomnio también sirve como factor de riesgo de suicidio. Actualmente, los Institutos de Medicina de los Estados Unidos estiman que por cada muerte por suicido entre los adolescentes, existen otros 100 adolescentes con intentos suicidas ${ }^{20}$.

Además del insomnio, la somnolencia diurna excesiva (SDE) es un problema frecuente que afecta a la población adolescente. Liu et al., en 2019, observaron que la 
SDE afecta aproximadamente al 29\% de los adolescentes y aumenta a medida que estos atraviesan la pubertad: se observa un incremento en la prevalencia del 19,8\% en la etapa Tanner 1 (prepubertad) y del 47,2\% en la etapa Tanner 5 (pospubertad). Además, el género femenino tiene mayor prevalencia desde la etapa Tanner 3 (media pubertad) ${ }^{21}$.

Vilela et al., en 2016, notaron que entre los factores que pueden incrementar el riesgo de sufrir somnolencia diurna excesiva se reporta la deprivación del sueño, que tiene una prevalencia del $39 \%$ en este grupo etario y aumenta con la edad. Se ha observado, asociación con hiperhidrosis del sueño y un cronotipo vespertino; síntomas de insomnio y uso de dispositivos electrónicos por más de una hora al día se asocian a la presencia de $\mathrm{SDE}^{22}$. Uno de los factores que más se relacionan con la SDE es la necesidad subjetiva reportada por los adolescentes de mayores horas de sueño, como lo observaron Ferrari et al..$^{23}$

Además, las enfermedades mentales y el alto estrés académico son factores de riesgo para desarrollar SDE. Luo et al. en 2018, observaron dentro de una población rural de adolescentes en China una asociación bidireccional entre la SDE y los síntomas de depresión y ansiedad, lo que puede indicar que existe una relación compleja entre las enfermedades mentales y las alteraciones del sueño en los adolescentes ${ }^{24}$.

Esto puede ocasionar en la vida de los adolescentes un menor desempeño académico, falta de desarrollo de la función cognitiva, uso de substancias y alteraciones en el humor, además de asociarse a problemas graves como el aumento de choques automovilísticos, que pueden llevar a la muerte ${ }^{25}$.

\section{Influencia del uso de los dispositivos móviles en los trastornos del sueño}

Los TIC han ganado popularidad entre la población joven sobre todo con el desarrollo de redes sociales, los dispositivos móviles y los videojuegos ${ }^{26}$. Sin embargo, su uso excesivo tiene repercusiones tanto a nivel físico como a nivel psicológico, llegándose a asociar con el consumo de drogas tales el como alcohol, tabaco o cannabis, bajo rendimiento académico y relaciones familiares conflictivas 5 . Se ha demostrado la relación entre el uso problemático del celular y los videojuegos con el evitar sentimientos negativos ${ }^{27}$.

Se han desarrollado escalas que permiten clasificar el uso de las TIC, entre las que se encuentran la escala MULTICAGE-TIC, creada a partir de la escala MULTICAGE-CAD para conductas adictivas y compulsivas y el cuestionario CAGE para alcoholismo, con el objetivo de adaptarse a las adicciones comportamentales modernas².

EI MULTICAGE-TIC está compuesto por veinte preguntas dicotómicas divididas en cinco escalas, y evalúa problemas relacionados al uso de móviles, internet, mensajería instantánea, videojuegos y redes sociales, permitiendo identificar aquellos usuarios con uso problemático de TIC. Este cuestionario ha demostrado valor predictivo para ítems acerca de problemas de atención, inestabilidad emocional, problemas emocionales y problemas inhibitorios en el comportamiento social. Por lo anterior, se considera una herramienta útil para identificar a aquellos usuarios con conductas problemáticas por el uso excesivo de dispositivos móviles u otras tecnología ${ }^{28,29}$.

Kuss et al., en 2016, definen el término «Uso problemático del internet» (UPI) como aquel que llega a interferir con las actividades cotidianas del usuario ${ }^{30}$, siendo la alteración del sueño una de las condiciones comórbidas más frecuentes. Se sugiere el uso nocturno de las TIC interfiere de manera directa en el ciclo circadiano y causa patrones de sueño irregulares, siendo el insomnio una de las principales consecuencias ${ }^{31}$.

Según Dube et al., en 2017, la presencia de tecnologías de entretenimiento y comunicación y su uso en la hora antes de dormir, se asocia negativamente a la duración, calidad y eficiencia del sueño, ocasionando la pérdida de 10,8 minutos de sueño para niños que usan celulares una hora antes de dormir, 10,2 minutos para niños que usan computadoras y 7,8 minutos para aquellos que utilizan la televisión una hora antes de dormir. Una deficiencia de 15 minutos de duración de sueño es suficiente para producir efectos clínicos y este límite disminuye cuando se utiliza más de un dispositivo. El mayor impacto es producido por el uso de celulares una hora antes de dormir, disminuyendo la calidad del sueño en $36 \%{ }^{32}$.

Johansson et al., en 2016, en una población de 259 adolescentes entre 13 y 21 años, observaron que el $97 \%$ hace uso de alguna tecnología una hora antes de dormir, el $47 \%$ utilizan tres o cuatro dispositivos antes de dormir y el $10 \%$ usan seis o más, y que el uso de múltiples dispositivos está asociado significativamente a una menor duración del sueño, sueño más ligero y despertar temprano. Determinaron mediante el uso de la escala de somnolencia de Epworth que el uso de internet antes de dormir se asocia de forma significativa con somnolencia diurna en exceso y la ausencia de sueño reparador $(\mathrm{Rho}=0,15 \text { a 0,31, } \mathrm{p}<0,05)^{33}$.

Según Hysing et al., en 2015, el uso de las TIC puede alterar el sueño al consumir el 
tiempo que normalmente se le dedicaría a este, causando alerta fisiológica mediante contenido estimulante o alterando el ciclo circadiano mediante la exposición a luz brillante ${ }^{34}$.

Además, el uso de las TIC durante la noche ha sido asociado a horas de dormir más tardías, pesadillas, sonambulismo y disminución de la capacidad regeneradora del sueño ${ }^{35}$. De mismo modo, Scott et al., en 2019, en una cohorte de 11872 adolescentes entre 13-15 años, determinaron que el 13,9\% hace uso de las TIC entre 3-5 horas diarias y el 20,8\%, más de 5 horas. Se asoció este uso excesivo con el inicio tardío del sueño con Odds Ratio (OR) de 2,14, vigilia en días escolares y problemas para volver a conciliar el sueño después de despertarse por la noche $(\mathrm{OR} 1,36)^{36}$.

Li et al., en 2017, en una muestra de 1015 estudiantes de secundaria buscaron la asociación entre la adicción al internet, adicción a redes sociales y la presencia de insomnio. La prevalencia de insomnio para la población en general fue de 37,2\%; sin embargo, para los sujetos con adicción a internet fue de $65,9 \%$ y con adicción a redes sociales de $54,1 \%$, obteniendo OR de 2,87 y 2,19, respectivamente. Los autores concluyen que existe una asociación significativa entre el uso prolongado de internet y la presencia de insomnio en adolescentes ${ }^{37}$.

\section{Influencia del confinamiento por COVID-19 en el uso de TIC y el patrón de sueño}

La COVID-19 y su rápida propagación ocasionaron que la Organización Mundial de la Salud (OMS) declarara una emergencia a la salud pública internacional en enero de 2020 y se establecieran cuarentenas rígidas mundiales en marzo. El cierre de las escuelas, aunado a la inseguridad económica, el distanciamiento social, la disminución de la movilidad y la preocupación por la pandemia, ha alterado las rutinas de niños y adolescentes, generando miedo, ansiedad, comportamientos agresivos o de desobediencia, aumentando además el riesgo de tendencias suicidas ${ }^{38}$.

A pesar que el uso excesivo de las TIC se asocia a diversos efectos negativos, la pandemia las ha vuelto indispensables e incluso beneficiosas, ya que las escuelas se han adaptado a plataformas en línea y los adolescentes pueden interactuar con sus pares por medio de redes sociales, videojuegos $u$ otras actividades interactivas. De acuerdo con Nagata et al., en 2021, el uso de las TIC podría representar una ventaja para lidiar con el distanciamiento social en aquellos niños y adolescentes familiarizados con es$\operatorname{tas}^{39}$.

Ying et al., en 2020, observaron que en niños entre 5 a 14 años que reciben clases en línea, 68,8\% de los padres reportaron 3 horas o más de uso de TIC, lo que excede el máximo recomendado de 2 horas. El 84,4\% reportaron menos de 2 horas de tiempo en exteriores $^{40}$. Drouin et al., en 2020, encontraron que los niños con niveles altos de ansiedad recurren con más frecuencia a las TIC y a las redes sociales, para interactuar con otros o informarse, mientras que niños de mejores capacidades socioeconómicas y con bajos síntomas de ansiedad recurrieron menos a estas ${ }^{41}$.

Durante el primer mes de confinamiento, Cellini et al., en 2020, reportaron un incremento de actividades que involucraba el uso de las TIC en las 2 horas previas al sueño, que se asoció a una mayor latencia del sueño y a un atraso de la hora de dormir. Además, las personas con síntomas de depresión, ansiedad y estrés, particularmente estudiantes y empleados, demostraron reducción de la calidad del sueño y desorientación temporal ${ }^{42}$.

Romero-Blanco et al., en 2020, compararon la cantidad y la calidad del sueño en estudiantes de enfermería previo a la cuarentena y después de esta, encontrando un incremento del tiempo pasado en cama y de la latencia del sueño, esta última relacionada al uso inadecuado de $\mathrm{TIC}^{3}$. Según Leone et al., en 2020, los efectos de la cuarentena en el sueño y el ciclo circadiano fueron mayores conforme disminuye la edad de los sujetos $^{43}$.

De acuerdo con Orben et al., en 2020, el aislamiento altera los patrones neurales en la sustancia nigra, ocasionando sentimientos de soledad y disminución de la felicidad. En adolescentes, el uso activo de redes sociales, con interacción directa con otras personas, puede aliviar los sentimientos asociados al aislamiento, provocar sentimientos de bienestar y dar acceso al apoyo social. El efecto del uso de las TIC en los adolescentes en confinamiento, por ende, será beneficioso si promueve la interacción y conexión directa con sus pares, mientras que actividades pasivas como ver fotos o videos, no ofrecen beneficio alguno ${ }^{44}$.

\section{Conclusiones}

El uso excesivo de tecnologías de la información y de la comunicación en la población adolescente, en especial horas antes de dormir, se relaciona con un patrón del sueño anómalo, que se identifica en una disminución en su calidad y cantidad. Se ha 
encontrado en la literatura que los trastornos del sueño más prevalentes en los usuarios de TIC son el insomnio y la somnolencia diurna excesiva.

\section{Agradecimiento}

A la Dra. Zairy García, nuestra tutora, por habernos apoyado en la elaboración de nuestro trabajo de investigación.

\section{Financiamiento}

Autor declara no tener fuente de financiamiento.

\section{Referencias bibliográficas}

1. Smartphone users worldwide 2020, Statista, Alemania, 2020. Disponible en: https://www. statista.com/statistics/330695/number-ofsmartphone-users-worldwide/

2. Cha S-S, Seo B-K. Smartphone use and smartphone addiction in middle school students in Korea: Prevalence, social networking service, and game use. Health Psychology Open; 2018, 5(1)1-15. DOI: $10.1177 / 2055102918755046$

3. Gupta R, Grover S, Basu A, Krishnan V, Tripathi A, Subramanyam A, et al. Changes in sleep pattern and sleep quality during COVID-19 lockdown. Indian J Psychiatry. 2020; 62(4): 370-378. DOI: 10.4103/psychiatry.IndianJPsychiatry $523 \quad 20$

4. Zhou S-J, Wang L-L, Yang R, Yang X-J, Zhang L-G, Guo Z-C, et al. Sleep problems among Chinese adolescents and young adults during the coronavirus-2019 pandemic. Sleep Medicine. 2020;74:39-47. DOI: 10.1016/j. sleep.2020.06.001

5. Muñoz-Miralles R, Ortega-González R, López-Morón MR, Batalla-Martínez C, Manresa $J M$, Montellà-Jordana N, et al. The problematic use of Information and Communication Technologies (ICT) in adolescents by the cross sectional JOITIC study. BMC Pediatrics. 2016;16(1):140-151. DOI: 10.1186/s12887016-0674-y

6. Hale L, Kirschen GW, LeBourgeois MK, Gradisar M, Garrison MM, MontgomeryDowns $\mathrm{H}$, et al. Youth Screen Media Habits and Sleep: Sleep-Friendly Screen Behavior Recommendations for Clinicians, Educators, and Parents. Child and Adolescent Psychiatric Clinics of North America. 2018;27(2):229-245. DOI: 10.1016/j.chc.2017.11.014.

7. Kim SY, Kim M-S, Park B, Kim J-H, Choi HG. Lack of sleep is associated with internet use for leisure. PLOS ONE. 23 de enero de 2018;13(1):e0191713. DOI: 10.1371/journal. pone. 0191713
8. Merino Andréu M, Álvarez Ruiz de Larrinaga A, Madrid Pérez JA, Martínez Martínez MÁ, Puertas Cuesta FJ, Asencio Guerra AJ, et al. Sueño saludable: evidencias y guías de actuación. Documento oficial de la Sociedad Española de Sueño. Rev Neurol. 2016;63(S02):1. DOI: $10.33588 / \mathrm{rn} .63502 .2016397$

9. Bruni O, Sette S, Fontanesi L, Baiocco R, Laghi F, Baumgartner E. Technology Use and Sleep Quality in Preadolescence and Adolescence. Journal of Clinical Sleep Medicine. 2015;11(12):1433-1441. DOI: 10.5664/ jcsm.5282

10. Puerto M, Rivero D, Sansores L, Gamboa L, Sarabia L. Somnolencia, Hábitos De Sueño Y Uso De Redes Sociales En Estudiantes Universitarios. Enseñ e Investig en Psicol. 2015;20(2):189195. Disponible en: https://www.redalyc.org/ articulo.oa?id=29242799009

11. Carter B, Rees P, Hale L, Bhattacharjee D, Paradkar M. Association between portable screen-based media device access or use and sleep outcomes: A systematic review and meta-analysis. JAMA Pediatrics. 2016; 170(12): 1202-1208. DOI: 10.1001/jamapediatrics.2016.2341

12. Zhang J, Paksarian D, Lamers F, Hickie IB, He J, Merikangas KR. Sleep Patterns and Mental Health Correlates in US Adolescents. J Pediatr. 2017; 182:137-143. DOI: 10.1016/j. jpeds.2016.11.007

13. Lira D, Custodio N. Los trastornos del sueño y su compleja relación con las funciones cognitivas. Rev Neuropsiquiatra. 2018; 81(1): 2028. DOI:10.20453/rnp.v81i1.3270

14. Jiménez-Genchi A, Monteverde-Maldonado E, Nenclares-Portocarrero A, et al. Confiabilidad y análisis factorial de la versión en español del índice de calidad de sueño de Pittsburgh en pacientes psiquiátricos. Gac Med Mex. 2008;144(6):491-496. Disponible en: https://www.medigraphic.com/pdfs/gaceta/gm-2008/gm086e.pdf

15. Miró E, láñez M, Cano-Lozano M. Patrones de sueño y salud. Revista Internacional de Psicología Clínica y de la Salud. 2002; 2 (2): 301-326. Disponible en: https://www.redalyc.org/ pdf/337/33720206.pdf

16. Guía de consulta de los criterios diagnósticos del DSM 5, Asociación Americana de Psiquiatría, Arlington Virginia, EE.UU, 2014. Disponible en: https://www.eafit.edu.co/ ninos/reddelaspreguntas/Documents/dsmv-guia-consulta-manual-diagnostico-estadistico-trastornos-mentales.pdf

17. de Zambotti M, Goldstone A, Colrain IM, Baker FC. Insomnia disorder in adolescence: diagnosis, impact, and treatment. Sleep Med Rev. 2018;39:12-24. DOI: 10.1016/j. smrv.2017.06.009

18. Sooyeon S, Cho N, Zhang J. Sex Differences in Insomnia: from Epidemiology and Eti- 
ology to Intervention. Curr Psychiatry Rep. 2018;20(9):69. DOI: $\underline{\text { 10.1007/s11920-018- }}$ 0940-9

19. Amaral MOP, de Almeida Garrido AJ, de Figueiredo Pereira C, Master NV, de Rosário Delgado Nunes C, Sakellarides CT. Quality of life, sleepiness and depressive symptoms in adolescents with insomnia: A cross-sectional study. Atención Primaria. 2017;49(1):35-41. DOI: 10.1016/j.aprim.2016.03.004

20. Bernert RA, Kim JS, Iwata NG, Perlis ML. Sleep Disturbances as an Evidence-Based Suicide Risk Factor. Curr Psychiatry Rep. Marzo de 2015;17(3):554. DOI: 10.1007/s11920-0150554-4

21. Liu Y, Zhang J, Li SX, Chan NY, Yu MWM, Lam SP, et al. Excessive daytime sleepiness among children and adolescents: prevalence, correlates, and pubertal effects. Sleep Med. 2019;53:1-8. DOI: 10.1016/j. sleep.2018.08.028

22. De Souza Vilela T, Bittencourt LRA, Tufik S, Moreira GA. Factors influencing excessive daytime sleepiness in adolescents. J Pediatr (Rio J). 2016;92(2):149-155. DOI: 10.1016/j. jped.2015.05.006

23. Ferrari Junior GJ, Barbosa DG, Andrade RD, Pelegrini A, Beltrame TS, Felden ÉPG. Subjective sleep need and daytime sleepiness in adolescents. Rev Paul Pediatr. 2019:37(2):209-216. DOI: 10.1590/19840462/;2019;37;2;00014

24. Luo C, Zhang J, Chen W, Lu W, Pan J. Course, risk factors, and mental health outcomes of excessive daytime sleepiness in rural Chinese adolescents: A one-year prospective study. J Affect Disord. 2018;231:15-20. DOI: 10.1016/i.jad.2018.01.016

25. Hansen SL, Capener D, Daly C. Adolescent Sleepiness: Causes and Consequences. Pediatr Ann. 2017;46(9):e340-e344. DOI: 10.3928/19382359-20170816-01

26. Matamala C. Uso de las TIC en el hogar: Entre el entretenimiento y el aprendizaje informal. Estud Pedagógicos Valdivia. 2016; 42 (3): 293-311. DOI: 10.4067/S0718$\underline{07052016000400016}$

27. García-Oliva C, Piqueras J. Experiential Avoidance and Technological Addictions in Adolescents. J Behav Addict. 2016; 5 (2): 293303. DOI: $10.1556 / 2006.5 .2016 .041$

28. Pérez E, de León J, Mota G, Luque M, Aguilar J, Alonso S, et al. Tecnologías de la Información y la Comunicación (TIC): abuso de Internet, videojuegos, teléfonos móviles, mensajería instantánea y redes sociales mediante el MULTICAGE-TIC. Adicciones. 2017;30(1):1932. DOI: 10.20882 /adicciones.806

29. Rodríguez M, Pedrero E, Rodríguez E, Fernández M, Pastor V, Mateo A, et al. MULTICAGE CAD-4 para la detección de adicciones conductuales: validez estructural después de la inclusión de una escala en el abuso de teléfonos inteligentes. An Psicol. 2019;35(1):41-6. DOI: 10.6018/analesps.35.1.324311

30. Kuss D, López O. Internet addiction and problematic Internet use: A systematic review of clinical research. World J Psychiatry. 2016; 6 (1): 143-76. DOI: 10.5498/wjp.v6.i1.143

31. Guo L, Luo M, Wang W-X, Huang G-L, Xu $Y$, Gao X, et al. Association between problematic Internet use, sleep disturbance, and suicidal behavior in Chinese adolescents. J Behav Addict. 2017;7(4):965-75. DOI: $\underline{10.1556 / 2006.7 .2018 .115}$

32. Dube N, Khan K, Loehr S, Chu Y, Veugelers P. The use of entertainment and communication technologies before sleep could affect sleep and weight status: a population-based study among children. Int J Behav Nutr Phys Act. 2017; 14 (1):97. DOI: 10.1186/s12966017-0547-2.

33. Johansson AEE, Petrisko MA, Chasens ER. Adolescent Sleep and the Impact of Technology Use Before Sleep on Daytime Function. J Pediatr Nurs. 2016; 31 (5): 498-504. DOI: 10.1016/j.pedn.2016.04.004

34. Hysing M, Pallesen $S$, Stormark KM, Jakobsen R, Lundervold AJ, Sivertsen B. Sleep and use of electronic devices in adolescence: results from a large population-based study. BMJ Open. 2015; 5 (1). DOI: 10.1136/bmjopen-2014-006748

35. Guo L, Luo M, Wang W, Huang W, Xu Y, Gao $X$, et al. Association between Information and Communication Technology Usage and the Quality of Sleep among School-Aged Children during a School Week. Journal of Behavioral Addictions 2018;7(4):965-975. DOI: $\underline{10.1556 / 2006.7 .2018 .115}$

36. Scott H, Biello SM, Woods HC. Social media use and adolescent sleep patterns: crosssectional findings from the UK millennium cohort study. BMJ Open. 2019; 9 (9): e031161. DOI: $10.1136 /$ bmjopen-2019-031161

37. Li J, Lau J, Mo P, Su X, Tang J, Qin Z, et al. Insomnia partially mediated the association between problematic Internet use and depression among secondary school students in China. J Behav Addict. 2017;6(4):554-63. DOI: $10.1556 / 2006.6 .2017 .085$

38. Oliveira W, Silva J, Andrade A, Micheli D, Carlos D, Silva M. A saúde do adolescente em tempos da COVID-19: scoping review. Cad Saúde Pública. 2020; 36 (8): e00150020. DOI: 10.1590/0102-311×00150020.

39. Nagata J, Abdel H, Gabriel K. Screen time for children and adolescents during the COVID-19 pandemic. Obes Silver Spring Md. 2020; 28 (9): 1582-1583. DOI: 10.1002/oby.22917

40. Zhao Y, Guo Y, Xiao Y, Zhu R, Sun W, Huang $W$, et al. The Effects of Online Homeschooling on Children, Parents, and Teachers of Grades 1-9 During the COVID-19 Pandemic. 
Med Sci Monit Int Med J Exp Clin Res. 2020;26: e925591-1-e925591-10. DOl: $\underline{\text { 10.12659/ }}$ MSM.925591

41. Drouin M, McDaniel B, Pater J, Toscos T. How Parents and Their Children Used Social Media and Technology at the Beginning of the COVID-19 Pandemic and Associations with Anxiety. Cyberpsychology, Behavior, and Social Networking. 2020; 23 (11): 727-736. DOI: $10.1089 /$ cyber.2020.0284

42. Cellini N, Canale N, Mioni G, Costa S. Changes in sleep pattern, sense of time and digital media use during COVID-19 lockdown in Italy. J Sleep Res, 2020;29:e13074. DOl: 10.1111/ jsr.13074
43. Leone M, Sigman M, Golombek D. Effects of lockdown on human sleep and chronotype during the COVID-19 pandemic. Curr Biol. 2020 Aug;30(16): R930-1. DOI: 10.1016/j. cub.2020.07.015

44. Orben A, Tomova L, Blakemore S. The effects of social deprivation on adolescent development and mental health. Lancet Child Adolesc Health 2020;4:634-40. DOI: $10.1016 /$ $\underline{\mathrm{S} 2352-4642(20) 30186-3}$ 